\title{
Comparative Analysis of the Physical Activity, Nutrition and Health Behaviour in Physical Education Students: Gender Differences
}

\author{
Popović Jasna ${ }^{1}$, Popović Miloš ${ }^{1}$, Popović Ružena ${ }^{2}$ \\ ${ }^{1}$ Faculty of Sport and Physical Education, University of Pristine/Kosovska Mitrovica, Leposavić \\ (Kosovo), Serbia \\ ${ }^{2}$ Faculty of Sport and Physical Education, University of Niš, Niš, Serbia \\ Email: ruzenapop@gmail.com
}

\begin{abstract}
A question that has interested physical activity researches in recent years is whether involvement in Physical Activity (PA) is associated with the adoption of other health behaviours, such as good nutrition and no smoking. In this pilot study was implemented the complex Diet Questionnaire, on PA, Health, Nutrition, within the group of the healthy adults, specifically selected students of the Faculty of Sport and PE (in Total 74 respondents). Applied $Q$ contains 7 segments (23 items) dealing with: Medical and Social status, Body mass, and Body High, Nutrition, Physical Activity, and Life Style. Study results are presented as comparative analysis of gender differences. Applied variables within (1-7) segments have non parametric properties. The proposed health outcomes suggest that considerable public health benefits could be achieved through PA. The identification of PA patterns is important if any effort to plan public health initiatives in this field.
\end{abstract}

Keywords: physical activity; nutrition; health behavior; gender differences

\section{Introduction}

Quality of life, as a term in medical literature, appeared more than 3 decades ago. Quality of life refers to the physical, physiological and social domains of health, seen as distinct areas that are influenced by e person's experiences, beliefs, expectations and perceptions. Today it is a very important issue in health care practice, as well as in scientific research. New era in medicine brings up interests not only in medical outcome of illness, but also in information about functioning, well-being, and other important health outcomes. Primary source of new information became standardized patient surveys in clinical population, and different measurement tools for new aspects of health, and new dimensions called quality of life have been developing since.

Although the idea of assessing the quality of life, using various measuring instruments is widely accepted by the researches of different areas, even yet has no unique definition. As a result, the indicators of the quality of life are not clearly defined, but include wide range of different measures, with a little difference between the scales for the assessment of the quality of life, and those for measuring overall health. However, many of authors observe quality of life as a complex function that contains more areas or domains of life and work of individuals, including physical and social function, mental status and type of symptoms, or health problems, and individual perception of health. (Popović, R., Samouilidou, E., Popović, J., \& Dolga, M., 2020).

On the occasion of the 100th Anniversary of the Olympic Games the scientist find that it is important to reaffirm the concepts of positive health postulated by Hypocrites and to reassess their relevance to the Olympic ideal and the health of the world's populations. 
The concept of positive health, as enunciated by Hypocrites, is based on the interaction of genetics, diet and physical activity. (Declaration of Olympia on Nutrition and Fitness, 1996).

A selective review of the previous studies. In the last years it has been a consistent goal of epidemiological research to identify specific behaviors that contribute to a variety of both negative and positive health outcomes, and to describe the underlying determinants of those behaviors. (Hansen, 2001). Health educators, in regard to improve health outcomes by implementing programs that attempt to eliminate risky behaviors and promote positive ones can then use such knowledge. (Orleans, Gruman, Ulmer, Emont, \& Hollendonner, 1999). A question that has interested physical activity researches in recent years is whether involvement in physical activity (PA) is associated with the adoption of other health behaviors, such as good nutrition and no smoking. In a review of the literature on leisure-time physical activity, elaborated by (Wankel, and Sefton, 1994) are point out on some relationships between PA and smoking behaviors (small negative), and no relationships between PA and alcohol consumption. A recent large-scale study of 2,400 Belgian adults, however, showed through cluster analysis that PA was not associated with other health behaviors (De Bourdeaudhuij, and Van Oost, 1999). A regular exercise program can enhance a preschooler's confidence, sharpen her motor skills and set a foundation for life-long habits. Researchers conclude that exercise alone is not enough to prevent obesity in young children. The study was based on a six-month trial involving more than 500 preschool-aged children. Youngsters in the exercise test group did boost their motor skills and exhibit improved behavior and self-esteem. However, past studies have also indicated that exercise programs must be accompanied by intensive diet and lifestyle change to offer any lasting benefits. (All authors mentioned are according to Moreno, Gonzalez-Gross, et al., 2007).

The study as by (Muhammad Riski Kurniawan, et al., 2020), uses a quantitative approach to the type of experimental research. Population and a sample of 30 students were selected by purposive sampling. Early childhood education (PAUD) is a form of education that focuses on laying the foundation for physical growth and development (fine and gross motor coordination), intelligence (thinking power, creativity, emotional intelligence, spiritual intelligence), social emotional (attitude and behavior as well as religion), language and communication, according to the uniqueness and stages of development that are passed by early childhood.

Another research uses a qualitative approach with descriptive analysis (Purnama, A., Yus, A., Wau, Y., 2020).This study aims to analyze The Profile of Fine Motor Development Achievement in Children of Island (5-6 Years Old) in Teulaga Tujuh Langsa Village. The data analyzed are the results of interviews by parents, and of children observations, and has developed in accordance with the 6 stages of achievement of fine motor development. Accomplishments include drawing according to patterns, imitating shapes, using stationery, cutting, sticking and expressing something with movement.

The purpose of this pilot study was to compare the Physical Activity habits, nutrition, and the health related lifestyle behaviors of college students from two diverse groups, regarding age and gender differences. It was assumed that meaningful comparisons were possible using existing instruments (Complex Diet Questionnaire/Weight management) with convenience samples of university students. This pilot study will provide data about nutrition knowledge, attitudes towards nutrition and the main determinants of food choice and preference, among male and female university students. 
The logical hypothesis was stated that significant age and gender differences would not be found, and that some small differences in health and nutritional lifestyle behaviors might be discussed, and partially understood in light of the macro level influences that seem to operate within the groups.

\section{Research Methods}

Subjects. Findings are based on a convenience sample of 74 students of the Faculty of Sport and Physical Education in Leposavic, University of Pristine (suited in Kosovska Mitrovica), and Republic of Serbia. The sample of examinees were not good labeled, regarding the age and gender specificity: 10 female PESs (13.5\%), and 64 male PESs (86.5\%), aged up 18-21 years (firs study year) to 25-30 years (third year od study).

Procedure. The instrument used in the study is the DIET Questioner for the assessment of the Physical activity, Nutrition and Health Behavior (according to Walker, Sechrist, \& Pender, 1987) and to compare the prevalence of selected behaviors in female and male, university students residing in Serbia. Pilot testing showed that chosen instrument is valid, reliable and applicable within population in our home country (Popovic R., et al., 2004). The students responses were collected during theoretical Outdoor activity lessons (first year of study), and on the Volleyball classes (third year of study). Applied Q contains seven segments (23 items) with possibility of choosing an alternative answers on: Medical History, Social History, Weight History, Diet History, Physical Activity, and Other, regarding the Life Style and Nutrition Quality of Life.

Statistical analysis. Student's responses to categorical questions were assessed, using the ratio of absolute $(\mathrm{n})$, and relative $(\%)$ frequency of statements, within provided items. Because of the large size and high complexity of the Q, it was not applicable to implement some advanced statistical procedures for the data analysis (some combination of parametric, and nonparametric data). The differences between female and male PESs responses were assessed and compared. Summary of Selected Anthropometric Variables (Body Mass, Body High), relative to Age-Scales and Groups Differences are presented in Table 1.

\section{Results and Discussion}

Table 1. Summary of Selected Variables (Body Mass, Body High) According to Age-Scales and Groups Differences: Female $(\mathrm{N}=10)$ and Male $(\mathrm{N}=64)$

\begin{tabular}{|c|c|c|c|c|c|c|c|c|}
\hline Sample & \multirow{2}{*}{\multicolumn{2}{|c|}{$\begin{array}{c}\text { Abs/Rel (\%) } \\
\text { Female (n=10) }\end{array}$}} & \multicolumn{2}{|c|}{ Abs/Rel (\%) } & \multicolumn{2}{|c|}{ Body Mass (0.1 kg) } & \multicolumn{2}{|c|}{ Body High $(0.1 \mathrm{~cm})$} \\
\hline Scale & & & & $(\mathrm{n}=64)$ & Fem & Male & Fema & Male \\
\hline Age & $\mathrm{f}$ & $\%$ & $\mathrm{f}$ & $\%$ & $\operatorname{kg}(\%)$ & $\mathrm{kg}(\%)$ & $\mathrm{cm}(\%)$ & $\mathrm{cm}(\%)$ \\
\hline 18-19 & 5 & $50 \%$ & 36 & 56.25 & $\begin{array}{l}51-60 \\
(20 \%),\end{array}$ & $\begin{array}{l}60-70 \\
(24 \%)\end{array}$ & $\begin{array}{c}163-167 \\
(40 \%)\end{array}$ & $\begin{array}{c}165-171 \\
(20 \%)\end{array}$ \\
\hline $20-23$ & 5 & $50 \%$ & 22 & 34.375 & $\begin{array}{c}61-65 \\
(60 \%),\end{array}$ & $\begin{array}{l}71-79 \\
(30 \%)\end{array}$ & $\begin{array}{c}172-185 \\
(60 \%)\end{array}$ & $\begin{array}{c}172-181 \\
(40 \%)\end{array}$ \\
\hline $24-30$ & - & - & 4 & 6.25 & $\begin{array}{l}66-70 \\
(20 \%)\end{array}$ & $\begin{array}{l}80-89 \\
(27 \%) \\
\end{array}$ & - & $\begin{array}{c}182-195 \\
(40 \%)\end{array}$ \\
\hline N/A & & blicable & 2 & 3.125 & - & $\begin{array}{l}90-100 \\
(19 \%)\end{array}$ & - & - \\
\hline
\end{tabular}

Nutrition Questionnaire (NCMC Adult Weight Management) 


\subsection{Medical History}

In determining the health status of PESs, within the first segment of Q, it was possible to make statements on individual, medical conditions of wide range (17 items). PESs refer only few health problems: High blood pressure (1/1.6\%), Asthma/Respiratory problems $10 \%$, 9 (3.25\%), and other: 9 (90\%), (81.25\%).

Considering data on medication use, the most female PESs (90\%) declare as no user, while $(10 \%)$ report occasionally use only for pain (Bactrim). A small number of male PESs refer as no user $(18 / 28.2 \%)$, only a few students $(2 / 3.2 \%)$ gave a positive answer, while the majority of students do not reply (44/68.75\%).

When considering the situation on the consumption of vitamins, minerals and other dietary supplements, the majority of female PESs do not replay (70\%), while only $30 \%$ indicated the consumption of Vitamin C, Magnesium and Aloe Vera. Male PESs (9/14.1\%) refer consummation of some type of supplement, other $(5 / 7.8 \%)$ do not use these products, and the most male students do not replay (50/78.1\%).

The majority of female PESs (90\%) do not comment on Allergy problems, while $(10 \%)$ are allergenic to dust, smoke and pollen. Male PESts have allergy discomfort to dust, smoke, pollen, nicotine, feathers, wasps/bees, mites (10/15.6\%), other students do not exhibit allergenic reactions (12/18.75\%), and majority do not reply (42/65.6\%).

In interpreting the results, only gender specificities were considered in relation to the actual sample of respondents. Recorded differences may be a starting point for identifying specific patterns of health behavior, but they cannot be applied as part of the design and implementation of a health education program targeted at the general population, since this study was conducted with a specifically selected sample of PESs, having a positive health status and higher level of physical abilities.

\subsection{Social History}

When is about nicotine use, great number of PESs students, $(\mathrm{F} / \mathrm{M})$, have declared as non-smokers $(80 \%),(50 / 78.1 \%)$, while others are not regular smokers $(20 \%)(14 / 21.9 \%)$, they report use up 5-10 (to 20) cigarettes in a typical day. When it comes to alcohol consumption, the most female PESs $(70 \%)$ do not drink alcohol, while other $(30 \%)$ report once a week, up 2 to 3-4 drinks at that point. The most male PESs (34/53.13\%) do not drink alcohol, while other (43/43.75\%) report (2-4) times a week (up 1 to10) drinks at that time. When declaring family status, or relationships to people who live with them: females PESs $(80 \%)$ are not married, live in the community, and other are married, or divorced (20\%). The marital status of male PESs is single (90\%), or married, no children (10\%).

\subsection{Weight History}

When declaring their own body weight, the most PES, both M/F, (80\%) have no concern about this, while the remaining $(20 \%)$ wanted to reduce BW. The majority $(90 \%)$ of the students do not reply to the question regarding the short and long term benefits of weight loss, but they gave information about current weight, current high, and Goal weight, then the Lowest adult weight, as well as the highest $\boldsymbol{B} \boldsymbol{W}$ that they have achieved in particular years of age. This data, without calculating Body Mass Index (BMI), does not provide a complete picture of student's body composition status. Likewise, a small percentage $(10 \%)$ of students, both $\mathrm{M} / \mathrm{F}$, think that reducing $\boldsymbol{B W}$ provides them with short-term satisfaction, while the other $(90 \%)$ do not reply. When it comes to expectations over the next two years, female PESs refer satisfaction, while male PESs have greater demands on increasing FA level 
and improving health. Regarding the absolute and relative ( $\mathrm{n} / \%)$ frequencies of responses by these items are given below.

Current weight (kg) (F): 51-60 (20\%), 61-65 (60\%), 66-70 (20\%); (M): 53-54 (2/3. 2\%), 6669 (11/17.2\%), 70-75 (13/20.3\%), 76-79 (12/18.7\%), 80-89 (17/26.5\%), 90-106 (8/12.5\%).

Current height (cm) (F): 163-167 (40\%), 172-185 (60\%); (M): 165-170 (5/7.81\%), 171-179 (12/18.7\%), 180-189 (48.4\%), 190-200 (12/18.75\%).

Goal weight (kg) (F): 52-60 (60\%), 62-68 (40\%); (M): 55-70 (7/10.9\%), 71-79 (13/20.3\%), $80-89(20 / 31.25 \%)$

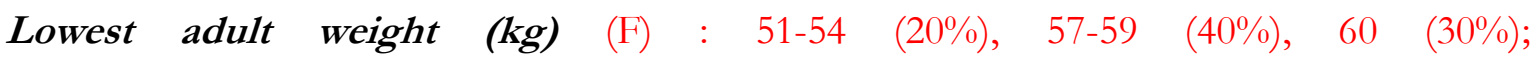
(M): 54-60 (7/10.9\%), 61-69 (10/15.6\%), $70-79$ (17/26.6\%), $80-90 \quad(8 / 12.5 \%), \quad 91-95$ (2/3.12\%), $90-99$ (8/12.5\%), 100-120 (4/6.25\%).

$\begin{array}{lllllllll}\text { Age/year at this weight } & (\mathrm{F}): & 14 & (10 \%), & 18 & (50 \%), & 19-20 & (30 \%) \text {; }\end{array}$ (M): $12-15$ (5/7.8\%), 16-19 (32/50\%), 20-25 (6/9.4\%), N/A (21/32.815\%)

Highest adult weight (kg) (F): 52 (10\%), 63-67 (70\%), $76 \quad$ (10\%); (M): $60-70$ (9/14.1\%), 71-79 (11/17.2\%), 80-89 (11/17.2\%), 90-100 (12/18.2\%), 101-120 $(3 / 4.7 \%), \mathrm{N} / \mathrm{A}(18 / 28.125 \%)$

Age at this weight (year) (F): $17-18 \quad(30 \%), \quad 19-20 \quad(50 \%), \quad 21 \quad(10 \%)$ (M): 15-19 (33/51.56\%), 20-30 (12/18.75\%), N/A (18/12/18.75\%)

\subsection{Diet History}

The most female PESs (90\%), and male (95.3\%), do not practiced a diet regiment, while F (10\%), and Male (3/4.69\%), stated they were dieting, not mentioned the type of diet. The most PESs F (90\%), M $(90.6 \%)$ do not try to reduce their BW in the past, while the remaining F (10\%), and M (3/4.69\%), practiced a diet regiment, and mentioned weight loss methods they tray: Female - fat burners, antioxidants, while Male opted for: moderate diet, increased training intensity, reduced calorie intake, physical activity, fat burners, avoiding dinner (6/9.4\%).

For a period of 6 months, PESs, both M/F, reduced their BW by $(2-6 \mathrm{~kg})$. They behave responsibly when it comes to proper nutrition, stating they do not try to control BW by using diet pills, laxatives, or avoiding foods, F (100\%), M (62/96.9\%). PESs do not answer the question of how much weight they have gained in the meantime! The most PESs, consume 67 times a week warm, served meals $\mathrm{F}(30 \%), \mathrm{M}(33 / 51.6 \%)$, home cooked food, with family members, $\mathrm{F}(50 \%), \mathrm{M}(47 / 73.4 \%)$, others less frequently, or consume restaurant food 1-2 times a week $\mathrm{F}(20 \%), \mathrm{M}(23 / 35.9 \%)$, or fast food/Takeaway $\mathrm{M}(3 / 4.7 \%)$.

The majority families traditionally consume their meals together (70\%), and in $30 \%$, meals are served at the same time. Female PESs do not answer the question regarding food allergy, nor do they indicate what foods have to avoid for any religious, personal or cultural reasons, nor did they answer the question regarding the recommendation of a physician to avoid certain foods.

Male PESs s gave a more detailed answer to the stated questions: some report that they are allergenic (10/15.6\%), the most do not comment this issue (49/76.6\%), while a few students $(5 / 7.8 \%)$ list some foods they do not consume due to digestive problems (lactose, 
mayonnaise, olives, fish, green beans, cabbage, liver). They do not cite barriers as for religious or cultural reasons $(6 / 9.375 \%)$, but the most avoid answering this question by $(58 / 90.6 \%)$.

\subsection{Physical Activity}

Physical activity, exercise and physical fitness. The detailed relationship between physical activity, fitness, fatness and health in adolescence remains to be clarified. Physical activity is a behavior that is, because of its complex nature, difficult to assess under free-living conditions. No single method is available to quantify all dimensions of the activity (total amount, intensity, frequency, duration, etc.). Lack of comparable data, especially regarding young individuals, facilitates the understanding of the complex relationship between these characteristics of physical activity, physical fitness and health outcomes.

This segment of the $\mathbf{Q}$, provides information on particular aspects of the PA, formulated in the form of self-explanatory questions. Tables $2 \mathrm{a}$ and $2 \mathrm{~b}$ summarize the numerical data collected, in regard to regular exercise, length of exercise or a given PA, and the extent and frequency of training during the week. No significant differences were observed when it came to PA. The most common answers, indicate regular PA, up 2-3, to 6-7 times per a week. Only 10\%-14\% of PESs do not comment on this issue. There is no gender difference in the length of practice for a particular PA or sport, the dominant response in both groups is $8-10$ years: $\mathrm{F}(30 \%), \mathrm{M}(31.25 \%)$.

Only $(10 \%)$ of female PESs do not regularly practice PA, nor answer the question regarding elective sport activity. Female students are regularly physically active (90\%), for a longer period of time (from 6 months to 16 years). They mainly exercise walking, running, strength training, sports (basketball, handball), tennis and karate (mostly recreational), but do not specify health restrictions regarding fitness for physical activity, which would be an impediment to increased exercise intensity.

Male PESs students gave a more detailed description of the activities they practiced: Fitness (10/15.6\%), Strength Training (8/12.5\%), Martial Arts, Karate/Kick box, (8/12.5\%), Endurance Run (8/12.5\%), Football (6/9.4\%), Sport Games - Basketball / Football / Handball (5/7.8\%), Basketball (4/6.2\%), Outdoor Activities - Walking / Cycling / Tennis / Street Boxing / Street Aerobics (4/4.7\%), and others do not replay (8/12.5\%).

\begin{tabular}{|c|c|c|c|c|c|}
\hline \multicolumn{6}{|c|}{ Table 2 a. Hove long are you involved (approximately) in Physical activity? } \\
\hline Sample & \multirow[t]{2}{*}{ Statements } & \multicolumn{2}{|c|}{ Females (10) } & \multicolumn{2}{|c|}{ Males (64) } \\
\hline Items & & Absolute (n) & Relative (\%) & Absolute (n) & Relative (\%) \\
\hline a) & 6-12 Months & 1 & $10(\%)$ & 15 & $23.4(\%)$ \\
\hline b) & 4-6 Years & 2 & $20(\%)$ & 15 & $23.4(\%)$ \\
\hline c) & $8-10$ Years & 3 & $30(\%)$ & 20 & $31.25 \%$ \\
\hline d) & 11-16 Years & 1 & $10(\%)$ & 5 & $7.8 \%$ \\
\hline e) & Not/Applicable & 3 & $30(\%)$ & 9 & $14.1 \%$ \\
\hline \multicolumn{6}{|c|}{ Table 2b. Approximately, how often do you exercise on weekly base? } \\
\hline a) & 2-3 time & 1 & $10(\%)$ & 12 & $18.75(\%)$ \\
\hline b) & 3-4 time & 2 & $20(\%)$ & 14 & $21.9 \%$ \\
\hline c) & 4-5 time & 2 & $20(\%)$ & 20 & $31.25 \%$ \\
\hline d) & 6 time & 2 & $20(\%)$ & 5 & $7.8 \%$ \\
\hline e) & Almost everyday & 2 & $20(\%)$ & 4 & $6.25 \%$ \\
\hline f) & Not/Applicable & 1 & $10(\%)$ & 9 & $14.1 \%$ \\
\hline
\end{tabular}




\section{a. Health barriers to participation in physical activity.}

In this segment of the Q, PESs were asked to mark all health barriers relative to participation in PA. According to the Note from the Q, if more offered answers are recorded, it would be necessary to schedule an appointment with a specialist doctor before continuing or increasing the intensity of exercise. As the good health status is "Conditio sine qua non" for FSPE study enrollment, students do not justify reply to these questions. PESs were expected not to have health problems that could be a barrier and limit their participation in exercise programs.

Health behaviors are difficult to measure, and this is certainly true regarding regular PA. Of particular concern is the accuracy of PA's self-reported evaluation. Factors that further impede obtaining accurate estimates include poor or incomplete response and inadequate sampling of time intervals during which PA is evaluated.

One of the major difficulties in determining the validity of PA measurements is the absence of an appropriate criterion, i.e., the "gold standard" for comparisons of measurements. In the absence of a proper assessment model, cardio-respiratory fitness is often used as a standard of validity for PA surveys. Because misclassification of physical activity could occur by using inappropriate measurement criteria, it could tend in biased studies to indicate no association, with a consistently established link, between physical activity and a lower risk of developing some diseases. Moreover, the researchers suggest that a more accurate measure of physical activity would likely contribute to even stronger associations with health. Thus, while measuring physical activity, by the currently available methods, is far from ideal, it provides a means of research and indicates important health benefits from physical activity.

\subsection{Other - Lifestyle}

\section{a. The importance of the need for a lifestyle change}

At the self-assessment scale, PESs declare the importance of the need to change individual lifestyle elements, with choice and strength of commitment to pre-prepared claims! The answers range up 1 to 10, with one (1) being less important, (5) partially important, (10) very important. A low score indicates that the person is very dissatisfied, and a high score indicates a very satisfied individual in terms of the lifestyle elements examined. Without a more adequate method of estimating the answers obtained, the authors of this study decided to collect points, representing the strength of the assignment, multiplied by the absolute frequencies (n) of the assertion, and divided by the number of respondents who provided the reply, than calculate the mean, and rank the strength of the assignment for the particular attitudes, especially for (Male/Female)! The frequency of a blank answers was not taken into account! The scale used in this segment of the $\boldsymbol{Q}$ includes some items that determine:

(a) The importance of lifestyle changes such as adjusting diet, increasing $P A$, and changing health-related behaviors? The strength of commitment is for F/M (6.3, 5.03) points F: 1(20\%), 5(30\%), 6(10\%), 10(40\%), M: 1(15/23.44\%), 4(1/1.56\%), 5(20/31.25\%), 7(1/1.56\%), 9(1/1.56\%), 10(11/17.19;

(b) The readiness to make lifestyle changes? the strength of commitment for $\mathrm{F} / \mathrm{M}(6.3,5.27)$ points F: 1(10\%), 5(50\%), 7(10\%), 10(30\%), M: 1(13/20.31\%), 5(23/35.94\%), 6(2/3.125\%), $9(1 / 1.56 \%), 10(12 / 18.75 \%)$;

(c) The certainty that lifestyle can be changed? the strength of commitment is at $\mathrm{F} / \mathrm{M}(\mathbf{6 . 6}, \mathbf{5 . 1 3})$ points F:1(10\%), 5(30\%), 9(10\%), 10(50\%), M: 1(15/23.35\%), 4(1/1.56\%), 5(21/32.81\%), 6(1/1.56\%), 8(1/1.56\%), 9(1/1.56\%), 10(12/18.75\%); 
(d) The lifestyle changes that FSPE students would be willing to do! The answers were as follows (F): Improving bealth (20\%), Physical activity/Sport (50\%), M: No need / none, undecided (9/14.0\%), Physical activity, Fitness, Weight loss, Sports, Miscellaneous (5/7.8125\%), Physical appearance, Everything needed, life needs, Health status, for the good of the family, education and confidence, miscellaneous $(10 / 15.625 \%)$.

(e) The aggravating circumstances for implementing a lifestyle change, PE students cite: (F) Lack of free time (20\%), Poor will / motivation (20\%), Family responsibilities (20\%), (M): No exist (6/9.4\%), Family Liabilities, Marriage, Condensed Schedule, College Degrees, Depression, Weakness, Financial Condition, Addiction, Miscellaneous (17/26.5\%).

\subsection{Nutrition Quality of Life}

Although the assessment of quality of life in recent years becomes more important in clinical and population based investigations, in designing of this type of studies numerous methodological questions are not solved. However, there is still no adequate answer on the question "who is the most appropriate person to be examined". Over 50\% of studies are based on adult men samples assessment. This is caused by belief that adults (especially men) are "the golden standard" in answering that question, bat in some situations the youth and women can be an optimal examinees subject.

This segment of $\boldsymbol{Q}$ (30 items) relate to the association of nutrition with other determinants of the quality of life: hygiene eating habits, knowledge of restrictive, "forbidden" foods, and satisfaction with one's appearance. PESs provided information regarding claims for a two-week memorization period (up the time of examination), and marked the answers as indicated below. First, the female positive answers are given as (\%), followed by the male answers, given as absolute and relative ( $\mathrm{n}, \%)$ frequencies. The number of non-replayed answers was not displayed. Order of items is by $\mathrm{Q}$ and the answers are:

PESs will consume enough food and are satisfied with the amount of it (50\%), (30/46.9\%); PESs consume a wide variety of foods $(80 \%),(21 / 32.8 \%)$; PESs have a feeling of bunger between meals (7/10.9\%); PESs will enjoy guilt-free foods $(20 \%),(2 / 3.1 \%)$; PESs will take sufficient time to personally select the best nutritional foods (40\%), (4/6.25\%); PESs will take the time to buy and prepare the best food for them (30\%), (5/7.8\%); PESs are satisfied with their appearance $(80 \%)$, (23/35.9\%); PESs are satisfied with the way they dress (80\%), (23/35.9\%); PESs express personal dissatisfaction with the consumption of foods not recommended (20\%), (5/7.8\%); PESs express their personal satisfaction with the time spent for themselves (50\%), (9/14.1\%); PESs express satisfaction with the successfully prepared meal (20\%), (5/7.8\%); PESs consume food as a personal reward (30\%), (4/6.25\%); PESs express a sense of happiness about the food consumed (30\%), (9/14.1\%); PESs express a feeling of anxiety that their food is "controlled" and "held in hand" PESs $(1 / 1.6 \%)$; PESs express the feeling that changing the food they consume would contribute to a better life (30\%), (3/4.9\%); PESs express frustration over food restrictions PESs (1/1.6\%); PESs - express anger over restriction on type and diet $(1 / 1.6 \%)$; PESs have a stressful situation with family/friends about the choice of food they consume (10\%), (2/3. 2\%); PESs friends/family resentment about the food they consume $(2 / 3.1 \%)$; PESs meet with others to exchange views and discuss nutrition issues (2/3.2\%); PESs have difficulty adhering to the recommended diet, while with family/friends $(50 \%)$, $(11 / 17.2 \%)$; (PESs) are aware of what type of food they should consume to lead a bealthy lifestyle $(40 \%)$, (14/21.9); (PESs) consider that they need to know the amount of food they can consume (20\%), $(11 / 17.2 \%)$ - believe that bealthy food choices should be made (10\%), (3/4.7\%); (PESs) consider that the recommended amounts of food should be consumed (50\%), (15/23.4\%); (PESs) consider that 
meals should be taken at appropriate intervals (10\%), (6/9.4\%); (PESs) feel that they should have a sense of confidence about avoiding "forbidden" foods (10\%), (3/4.7\%).

Nutrition and physical activity interact in harmony and are the two most important positive factors that contribute to metabolic fitness and health interacting with the genetic endowment of the individuals. Genes define opportunities for health and susceptibility to disease, while environmental factors determine which susceptible individuals will develop illness. Therefore, individual variation may need to be considered to achieve optimal health and to correct disorders associated with micronutrient deficiency, dietary imbalance and a sedentary lifestyle. Every child and adult needs sufficient food and physical activity to express their genetic potential for growth, development, and health. Insufficient consumption of energy, protein essential fatty acids, vitamins (particularly vitamins A, C, D, E and B complex) and minerals (particularly calcium, iron, iodine, potassium and zinc), and inadequate opportunities for physical activity impair the attainment of overall health and muscle-skeletal function.

Balancing physical activity and good nutrition for fitness is best illustrated by the concept of energy intake and output. For sedentary populations, physical activity must be increased; for populations engaging in intense occupational and/or recreational physical activities, food consumption may need to be increased to balance their energy needs. Nutrient intakes should match more closely human evolutionary heritage. The choice of foods should lead to a diverse diet high in fruits and vegetables, and rich in essential nutrients, particularly protective antioxidants and essential fatty acids.

The current level of physical activity should much more closely our genetic endowment. Reestablishment of regular physical activity into everyday life on a daily basis is essential for physical, mental, and spiritual well-being. For all ages and both genders the physical activity should be appropriately vigorous and of sufficient duration, frequency, and intensity using large muscle groups rhythmically and repetitively. Special attention for adequate nutrition should be given to competitive athletes.

The attainment of metabolic fitness through energy balance, good nutrition and physical activity, reduces the risk of and forms the treatment framework for many modern lifestyle diseases such as diabetes mellitus, hypertension, osteoporosis, some cancers, obesity, and cardiovascular disorders. Metabolic fitness maintains and improves muscle-skeletal function, mobility, and the activities of daily living into old age.

Education regarding healthy nutrition and PA must begin early and continue throughout life. Nutrition and physical activity must be interwoven into the curriculum of school age children and of educators, nutritionists and other health professionals. Positive role models must be developed and promoted by society and the media. Major personal behavioral changes supported by the family, the community, and societal resources are necessary to reject unhealthy lifestyles and to embrace an active lifestyle and good nutrition.

National governments and the private sector must co-ordinate their efforts to encourage good nutrition and physical activity throughout the life cycle and thus increase the pool of physically fit individuals who emulate the Olympic ideal. The ancient Greeks/Helens attained a high level of civilization based on good nutrition, regular physical activity, and intellectual development. They strove for excellence in mind and body. Modern men, women, and children can emulate this Olympic ideal and become swifter, stronger, and fitter through regular physical activity and good nutrition (Declaration of Olympia on Nutrition and Fitness, 1996). 


\section{Conclusion}

When it is about health related behaviors, states/members of EU, yet in 1995th have harmonized agreement on the necessity of important development in domain of positive health habits, including good balanced diet, avoiding of nicotine use, appropriate PA, and good stress management, positive effect on mental health, and psychological status in sense of reduction of fears, and stress reactions.

As such, there is growing interest in the macro level determinants of health behavior, including the role of social norms, culture, mass media, national health policies, advertising practices, physical and social environments. Comprehensive behavior change interventions of the future will likely be most effective if they are able to address, both, micro and macro level determinants of behavior. However, the impact of public health is depended on people being physically active. The identification of PA patterns is important if any effort to plan public health initiatives in this field.

Given the above-mentioned problems, this pilot study group plans to describe in the future the nutritional status project of the Serbian students, and to improve health-related nutritional aspects by proposing an innovative educational intervention and developing new healthy foods attractive for adolescents, youth and young adults.

New and modern tools for health promotion need to be developed focusing on this specific population and considering gender differences. Health promotion should not force models of behavior onto individuals or groups. They need a food culture based on foods to eat, rather than foods to avoid, and understanding of suitable weight-control measures.

Computer-tailored nutrition and PA education is an innovative, promising and costeffective tool to motivate people to make healthy dietary and PA changes. It provides respondents with individualized feedback about dietary behavior and PA. The available evidence indicates that computer-tailored education is more effective in motivating people to make changes than general nutritional and physical activity education.

In summary, there are insufficient data to draw any conclusions about the nutritional quality and diets of the university students in Serbia. The Pilot study has not provide harmonized and comparable data on food intake among male and female PE students. For this purpose, authors proposed to use in the future study an existing tool called YANA-C (Young Adolescents' Nutrition Assessment on Computer) which has to bee adapted to local conditions for all participating age and gender different groups, using a standardized protocol.

\section{Limitations}

The main gaps in the knowledge concerning the nutritional status situation in university PE Students are: lack of harmonized and comparable data on food intake; lack of understanding regarding the role of eating attitudes, food choices and food preferences; lack of harmonized and comparable data on PA, and physical fitness; lack of comparable data about obesity prevalence and body composition; and lack of comparable data about micronutrient status.

The present dietary data on university students are flawed with serious methodological problems on food and nutrient intake. For instance, various collection methods are used. Therefore, there is a need for harmonization and standardization of methods to be used in nutrition surveys in specific selected samples of university students. 
Despite the intuitive appeal of education as a means of improving diet, many studies in this area have failed to find significant associations between nutritional knowledge (Cognitive determinants of eating habits) and dietary behavior. Since the way in which nutrition knowledge transforms into dietary behavior, and nutrient intake may vary among populations, it appears important to assess whether nutrition knowledge is associated with particular food choices and nutrient intakes before any nutrition intervention is initiated in a given population.

\section{References}

Boreham, C., Twisk, J., Neville, C., Savage, M., Murray, L., Gallagher, A. (2002). Associations between physical fitness and activity patterns during adolescence and cardiovascular risk factors in young adulthood: The Northern Ireland Young Hearts Project. International Journal of Sports Medicine; 23(1): 22-6.

Brug, J., Oenema, A., Campbell, M. (2003). Past, present and future of computer-tailored nutrition education. American Journal of Clinical Nutrition; 77(4): 1028S-34S.

Contento, I., Balch, G.I., Bronner, Y.L. (1995). The effectiveness of nutrition education and implications for nutrition education policy, programs and research. Journal of Nutrition Education; 13: 1-11.

Craig, C.L., Marshall, A.L., Sjostrom, M., Bauman, A.E., Booth, M.L., Ainsworth, B.E., et al. (2003). International physical activity questionnaire: 12-country reliability and validity. Medicine and Science in Sports and Exercise; 35: 1381-95.

Dallongeville, J., Marecaux, N., Cottel. D., Bingham, A., Amouyel, P. (2000). Association between nutrition knowledge and nutritional intake in middle-aged men from Northern France. Public Health Nutrition; 4: 27-33.

De Bourdeaudhuij, I., Sallis, J., Vandelanotte, C. (2002). Tracking and explanation of physical activity from adolescence to young adulthood. A 7-year prospective study. Research Quarterly for Exercise and Sport; 73: 376-85.

Declaration of Olympia on Nutrition and Fitness (1996). The Third Conference on Nutrition and Fitness, International Panel (Chair: A.P. Simopoulos), Athens, Greece.

Hansen, W. (2001). The future of health behavior and prevention research: What will change in the next 25 years. American Journal of Health Behavior, 25(3), 228-233.

Hasselstrom, H., Hansen, S.E., Froberg, K., Andersen, L.B. (2002). Physical fitness and physical activity during adolescence as predictors of cardiovascular disease risk in young adulthood. Danish youth and sports study. An eight-year follow-up study. International Journal of Sports Medicine; 23(1): 27-31.

Janz, K.F., Dawson, J.D., Mahoney, L.T. (2000). Tracking physical fitness and physical activity from childhood to adolescence: The Muscatine Study. Medicine and Science in Sports and Exercise; 32: 1250-7.

Janz, K.F., Dawson, J.D., Mahoney, L.T. (2002). Increases in physical fitness during childhood improve cardiovascular health during adolescence: the Muscatine Study. International Journal of Sports Medicine; 23(1): 15-21.

Moreno, Gonzalez-Gross, Kersting, Molncír, de Henauw, et al. (2007). Assessing, understanding and modifying nutritional status, eating habits and physical activity in European adolescents: The HELENA (Healthy Lifestyle in Europe by Nutrition in Adolescence) Study. Public Health Nutrition: 11(3), 288-299.

Muhammad Riski Kurniawan, Syamsu lrizal, Razali, Israwati (2020). The Ability of Gymnastics Motoric Perceptual Based on Local Culture for Early Childhood in Aceh Province. Budapest International Research and Critics in Linguistics and Education (BirLE), Volume (3):749-762, e-ISSN: 2655-1470, p-ISSN: 2655-2647.

Nowak, M. (1998). The weight-conscious adolescent: body image, food intake, and weightrelated behavior. Journal of Adolescent Health; 23: 389-98. 
Orleans, C.T., \& Cummings, K. (1999). Population-based tobacco control: progress and prospects. American Journal of Health Promotion, 14(2), 83-91.

Popovic, R., Muratidou, E., \& Kocic J. (2004). A cross-cultural comparison of health promoting behaviors among females. Book of Abstracts (pp. 166-167), "2nd FIEP European Congress", Vrnjačka Banja, Serbia \& Montenegro.

Popović, R., Samouilidou, E., Popović, J. \& Dolga, M. (2020). Assessment of the Quality of Life, Health, and Social Wellness in Upper Elementary School Students: CrossCultural and Gender Specificity. Britain International of Humanities and Social Sciences (BIoHS), Volume 2(1): 127-142. https://doi.org/10.33258/biohs.v2i1.158

Purnama, A., Yus, A., Wau, Y. (2020). The Profile of Fine Motor Development Achievement in Children of Island (5-6 Years Old) in Teulaga Tujuh Langsa Village. Budapest International Research and Critics in Linguistics and Education (BirLE), Volume 3(1): 127-132 e-ISSN: 2655-1470, p-ISSN: 2655-2647.

Sjostrom, M., Ekelund, U., Yngve, A. (2004). Assessment of physical activity In: Gibney, M.J., Margetts, B.M., Kearney, J.M., Arab, L., eds. Public Health Nutrition. The Nutrition Society Textbook Series. Oxford: Blackwell Publishing; 2004, pp. 83-105.

Vereecken, C.A., Covents, M., Matthys. C., Maes, L. (2005). Young adolescents' nutrition assessment on computer (YANA-C). European Journal of Clinical Nutrition; Volume 59: 658-67.

Wardle, J., Parameter, K., Waller, J. (2000). Nutrition knowledge and food intake. Appetite; Volume 34: 269-75.

Walker, S.N., Sechrist, K.R., \& Pender, N.J. (1987). The health-promoting life style profile: Development and psychometric characteristics. Nursing Research, 36(2): 76-81. 